\title{
Objectif littératie(s) : pour de nouvelles modélisations en didactique des langues étrangères
}

\author{
Christine Sagnier \\ Department of French and Italian, Université de Princeton \\ csagnier@princeton.edu
}

\section{Forces de renouvellement}

Alors que nos universités aux Etats-Unis reconnaissent les nécessités de réforme des cursus de langue, littérature et culture et qu'émergent de nombreuses interrogations sur les approches et structures à mettre en place pour répondre aux objectifs et besoins institutionnels ainsi qu'aux profondes mutations du champ professionnel, l'heure est au bilan des décennies précédentes. Les remises en cause des découpages institutionnels et des missions des départements renvoient à des questionnements didactiques concernant les pratiques dominantes et soulèvent l'intéressante question du renouveau des méthodologies existantes et des formations des futurs enseignants universitaires.

Il est évident que les ensembles pédagogiques mis en place aujourd'hui pour les langues étrangères dans la plupart des universités aux Etats-Unis ne sont pas satisfaisants et que la fracture entre « enseignement de la langue » et « enseignement de la littérature et culture », renforcée par les structures institutionnelles et par les approches didactiques dont les objectifs semblent parfois contradictoires, a contribué à affaiblir les possibilités de développement des départements, comme le note le rapport de la commission d'experts de la Modern Language Association (MLA), publié en $2007^{1}$.

Redéfinir la mission centrale d'un département, pour retrouver une cohérence globale et des objectifs communs, implique de se poser la question des cursus et de leurs contenus, mais aussi celle de la formation. Pour que le renouveau se fasse, il semble impératif que la réflexion didactique porte sur les systèmes en place et que soit fait un réel effort pour repenser les formations des doctorants en langue, littérature et culture (LLC), afin de doter les générations de jeunes enseignants-chercheurs à venir d'un véritable bagage professionnel. Or, par manque de temps, de questionnement didactique adéquat, sous la pression des contraintes horaires, les formations initiales à l'université sont rarement adéquates en tant que préparation à de véritables compétences professionnelles. Comme le souligne le rapport de la MLA, les séparations artificielles entre programme de langue et programme de littérature, établies dans les années 70 , ont généré et véhiculé des représentations associant l'activité de recherche à la littérature et celle de la pratique et de la pédagogie à l'enseignement de la langue, créant ainsi une opposition réductrice qui a fait obstacle à l'essor d'une réelle réflexion sur la didactique des langues étrangères (DLE) aux Etats-Unis, qui engloberait la problématique de l'enseignement littéraire.

Le rapport de la MLA pose des jalons importants, puisqu'il propose une vision renouvelée de la mission centrale de nos départements LLC. L'approche privilégiée est en effet définie en ces termes: «a broad, intellectually driven approach to teaching language and culture in higher education », rejetant la polarisation entre l'approche «fonctionnelle » souvent trouvée au premier niveau du cursus et l'approche «humaniste» typique des niveaux supérieurs. L'objectif declaré est de former, dans l'enseignement supérieur, à la lumière des événements tragiques de septembre 2001, de nouvelles générations d'étudiants, qui seraient moins ethnocentriques, plus ouverts sur d'autres cultures, dotés de compétences linguistiques et translinguistiques, mais aussi de véritables compétences culturelles et transculturelles. La capacité à opérer dans des systèmes culturels différents, à saisir les enjeux, les valeurs et les raisonnements prédominants au cœur d'une culture, à comparer les perspectives pour tenter de comprendre «l'Autre » requiert donc la mise en place de nouveaux dispositifs. 
L'idée de la sensibilisation et de la rencontre avec l'altérité à travers l'étude d'une langue et d'une culture étrangère n'est pas nouvelle, nous ne reviendrons pas sur l'historique de la notion. Ce qui est intéressant, c'est de pousser la réflexion au-delà de simples déclarations concernant les compétences linguistiques, translinguistiques et transculturelles. Si ces objectifs constituent le cœur de la mission des départements, comment mettre en place les modalités qui permettent de les atteindre et comment s'assurer que se développent des cursus unifiés et des formations d'enseignants-chercheurs qui stimuleraient l'innovation didactique et l'évolution des méthodologies?

De nombreux chercheurs en didactique des langues (DLE) ont contribué à la réflexion en matière de compétences culturelles ou «transculturelles » et une quantité importante de travaux, souvent regroupés sous l'appellation de «literacy approach », (que nous traiterons plus en détail dans la partie suivante) ont été publiés, sur la question de l'intégration des apprentissages linguistiques et culturels. Les idées qui y sont développées sont très riches et potentiellement porteuses de multiples possibilités, particulièrement pour les niveaux intermédiaire et avancé de l'apprentissage linguistique, mais l'étude de ces propositions reste trop souvent confinée aux spécialistes du domaine et ne se diffuse pas assez largement dans les départements où sont formées les futures générations d'enseignants-chercheurs en LLC.

Pour que ces travaux puissent nourrir la réflexion didactique et contribuer à l'élaboration de nouveaux ensembles pédagogiques et pratiques de classe dans l'enseignement supérieur, pour réduire la fracture entre les premiers niveaux du cursus et les niveaux supérieurs, il faut que les didacticiens s'attachent à clarifier un grand nombre de notions-clés et à mettre en évidence les passerelles et les correspondances au sein d'un cadre théorique suffisamment large pour que les articulations interdisciplinaires émergent de façon plus nette. Savoir construire des objets d'apprentissage pour les futurs enseignants-chercheurs et créer des formations les dotant de réels outils professionnels serait le prérequis à toute espoir d'évolution à long-terme de nos dispositifs et de nos approches.

Il semble aujourd'hui que nous ayons atteint ce point, dont parle Christian Puren, (1999), où se conjuguent des forces multiples pour le renouvellement: constat des limites d'une méthodologie, nouvelles attentes sociales et politiques, nouveaux apports théoriques, innovations technologiques, tout semble se conjuguer pour que dessinent de nouvelles configurations dans le domaine de la DLE aux Etats-Unis. La balle est dans le camp des didacticiens prêts à relever les défis.

\section{Des défis importants}

Les obstacles sont nombreux et se situent principalement à deux niveaux : tout d'abord à celui des institutions, où il y a un manque criant de spécialistes de DLE pour faire un "état des lieux » des programmes dans leur ensemble et proposer de nouveaux dispositifs. En effet, en raison des découpages institutionnels de la plupart des universités, très peu de spécialistes de linguistique appliquée et didactique se trouvent en poste dans des départements de LLC, où les possibilités d'évolution de carrière, de promotion ou de titularisation sont rares pour ces spécialités. De façon générale donc, ces spécialistes migrent vers les départements de Sciences de l'Education, de Linguistique ou d'Anglais Langue Etrangère et leurs réflexions n'alimentent donc pas les pratiques sur le terrain en LLC.

Le deuxième obstacle est la fragmentation actuelle de la discipline. Le champ de la didactique des langues étrangères est en effet aujourd'hui traversé par de nombreux courants, ce qui témoigne du dynamisme de ce domaine, mais constitue une sorte de «nébuleuse » difficilement décodable par les non-initiés. Pour que se dessinent de nouveaux espaces de dialogues et que se mette en place une articulation solide entre premiers et derniers niveaux dans l'enseignement supérieur, il semble essentiel de procéder à une remise à plat des approches, à un travail de clarification conceptuelle et d'intégration des paradigmes de recherche en DLE, à la lumière des objectifs proposés. Ces efforts seraient bénéfiques à la fois pour les cursus et pour la mise en place de nouvelles formations pour les doctorants de LLC.

Au niveau des cursus, le cadre de référence dans les premiers niveaux est largement celui de l'Approche Communicative (AC), dont on connaît les limites et les zones d'ombre, nous y reviendrons, alors que dans les niveaux supérieurs règne l'éclectisme en matière d'approche didactique : on y privilégie en effet 
plus souvent les contenus que la forme linguistique et les démarches ne sont que trop rarement intégratives. Ces ruptures et fractures en termes de cursus et d'approche rendent les tentatives de mise en place de formation pour les futurs enseignants-chercheurs particulièrement difficiles sur le terrain. De nombreux doctorants en formation ont en effet des difficultés à imaginer des possibilités de cursus unifié et adhèrent donc souvent à la perspective duelle caractéristique de la plupart de nos départements, selon laquelle l'enseignement-apprentissage de la langue est déconnecté de celui de la littérature. Formés de façon plus ou moins empirique, sans réelle réflexion critique sur les approches didactiques, ces futurs enseignants se fabriquent des méthodologies au fil de leur expérience, reproduisant souvent les schémas existants, sans que soit rendue explicite la possibilité d'une autre vision.

Pour nous, en réponse aux objectifs recommandés par la Commission de la Modern Language Association, la voie vers un cursus plus riche et plus unifié, qui proposerait « content from the beginning, language to the end », passe par la réflexion didactique, ainsi que par la mise en place de réelles formations professionnelles pour les futurs enseignants. Sans changement, nous risquons de voir nos programmes de littérature étrangère se dissocier de plus en plus des apprentissages linguistiques (de nombreuses universités proposent en effet déjà des cours de littérature en anglais dans le cadre de cursus de LLC) et nos programmes de langue adopter une approche de plus en plus fonctionnaliste, ce qui rendrait difficile la réalisation d'objectifs tels que la compétence «translinguistique et transculturelle » prônée par la Commission.

Ces objectifs ambitieux, nous l'avons mentionné, ont fait l'objet de nombreuses propositions de la part de différents chercheurs, que nous avons regroupés sous le terme de «literacy approach ». Que signifientils? Pour Kern (2000), les programmes linguistiques doivent promouvoir la littératie et préparer de futures générations d'étudiants à l'internationalisation et la mondialisation croissante de nos sociétés. Le terme «littératie » renvoie, dans sa définition minimale, à la capacité de lire et d'écrire, puis à une certaine familiarisation avec la littérature. On retrouve ici les buts que se fixent généralement la plupart de nos départements de LLC. Mais c'est une vision plus large de cette notion que proposent les chercheurs du courant « littératie ». Ils la définissent en effet comme « une capacité à produire et à interpréter des textes, une conscience critique des relations entre textes, conventions discursives et contexte culturel et social » (Kern, 2000, p. 6). Cet auteur suggère même que le terme soit utilisé au pluriel, les multiples «littératies» étant définies comme des pratiques d'utilisation, de production et d'interprétation dynamiques, situées culturellement et historiquement, de textes écrits et oraux, pour répondre à des objectifs sociaux » (Kern , 2000, p. 6). Une centration sur la littératie, qui considèrerait les activités d'écriture et de lecture dans leur contexte social d'utilisation permettrait une discussion plus unifiée des relations entre auteurs, textes, culture et apprentissage linguistique. La notion de «texte » est ici aussi prise au sens large, puisqu'il s'agira de formes diverses de "récits» qui émergent d'une culture et incluent par exemple la publicité, les discours politiques, les articles journalistiques, scientifiques, les chansons, films ou vidéos musicales, etc.

L'objectif « littératie » élargit les perspectives sur les relations entre lecture et écriture et peut fournir, comme le soutient Kern, un principe organisateur pour les programmes de LLC, qui favoriserait la mise en place de modalités d'enseignement plus cohérentes au sein d'un cursus et d'un niveau à l'autre. Adopter cet objectif signifie encourager la réflexion critique et le développement de compétences linguistiques, translinguistiques et transculturelles à tous les niveaux du cursus. Cette approche et la recherche d'un équilibre dans nos cursus entre « forme » et « contenus » exigent une mise à plat de nos pratiques qui ne pourra s'effectuer sans clarification conceptuelle en didactique. C'est une reconceptualisation et une démarche d'articulation théorique en DLE qui contribueront à mettre en évidence les correspondances et passerelles possibles entre les approches actuelles apparemment incompatibles de nos cursus de langue et de littérature.

\section{Conceptualisations et passerelles théoriques}

Notre objectif ici ne sera pas de passer en revue tous les différents courants pour examiner ce qu'ils peuvent apporter, mais de nous concentrer sur deux approches récentes et de montrer comment la mise en 
évidence de l'existence de passerelles théoriques peut nourrir la réflexion sur l'élaboration de cursus de LLC pour l'enseignement supérieur et aider à la création de formations professionnalisantes pour les futurs enseignants-chercheurs. Au cœur de nos préoccupations se trouve la rupture méthodologique actuelle entre les premiers et derniers niveaux de nos programmes. Nous argumenterons donc à la fois pour une clarification conceptuelle en didactique, mais aussi pour une démarche intégrative, qui rendraient plus explicites les correspondances théoriques, encouragerait la réflexion critique et permettrait de trouver des solutions innovantes pour répondre aux problèmes actuels.

Il ne s'agira pas ici de renier les apports de l'AC, ni de négliger la dimension communicative du langage, mais plutôt d'aller au-delà de ses propositions et de dégager des pistes de réflexion pour l'avenir. Nous nous concentrerons sur l'intérêt que présentent deux grands courants trop peu souvent mis en regard, celui des travaux portant sur les stratégies d'apprentissage et la métacognition (que nous regrouperons pour l'heure sous l'appellation de "modèles métacognitifs ») et celui qui émane des recherches d'inspiration vygotskienne, que nous qualifierons pour l'heure, par souci de simplification, de «cadre socioculturel et socio-interactionniste», en référence aux recherches publiées dans le domaine anglo-saxon (sociocultural theory), mais également aux remarquables travaux effectués par les équipes francophones de l'Université de Genève (interactionnisme socio-discursif). Ces deux courants considèrent en effet l'individu apprenant comme un acteur social, qui développe des compétences grâce à ses interactions avec d'autres acteurs sociaux. Nous essaierons d'identifier brièvement les points de convergence et d'articulation et nous plaiderons donc pour que se développent des espaces de dialogue dans un cadre théorique qui serait celui d'une « cognition située », qui tiendrait compte à la fois des apports des recherches sur le traitement de l'information par le sujet apprenant et des dynamiques et processus sociaux et historiques qui structurent et influencent les apprentissages. Nous nous intéresserons aussi à la compatibilité de ces deux courants avec l'AC, dans un objectif de recherche de nouvelles approches méthodologiques pour l'élaboration de cursus dans nos programmes de LLC, particulièrement en ce qui concerne la lecture et la production écrite en langue étrangère (LE).

Nous avons déjà évoqué la prédominance de l'approche communicative dans les premiers niveaux de l'apprentissage. De nombreux auteurs ont analysé en détail les forces et les faiblesses de l'approche communicative (Cuq et Gruca, 2005 ; Moirand, 1990 ; Puren, 1999) et nous nous contenterons ici d'une brève synthèse pour la question qui nous intéresse. Les méthodologies communicatives ne répondent en effet que partiellement aux objectifs des programmes universitaires. Pour le débat qui nous préoccupe, celui de l'élaboration de nouveaux cursus plus unifiés et ciblant le développement de solides compétences linguistiques, translinguistiques et transculturelles, on peut se demander avec Swaffar et al. (1991), comment la pratique visant à parler d'objets concrets liés à la vie quotidienne et la domination de l'oral, proposées dans les premiers niveaux sous l'influence de l'AC, peuvent préparer efficacement les apprenants à s'exprimer et à évaluer des idées et des concepts abstraits dans la langue-cible, sans que soient mis en place des démarches visant à développer l'émergence de telles compétences. Dans nos programmes, tout se passe comme si seule la progression linguistique permettait ce passage entre traitement d'informations factuelles et capacités à analyser, à élaborer des arguments, à interpréter des productions émanant de la culture-cible. Or, cette transition n'est pas évidente et requiert des méthodologies spécifiques. Nous argumenterons donc, avec Kramsch $(1993,1995)$ pour « une pédagogie de pratique interprétative », ce qui nous amènera à proposer une démarche intégrative qui se situera au croisement des théories sociocognitives et socioculturelles et plaidera pour une intégration conceptuelle permettant l'établissement d'un dialogue constructif entre les divers paradigmes de recherche en DLE. Il s'agira de se poser la question des démarches à mettre en œuvre pour répondre à des objectifs de littératie, pour que soient mieux intégrés les apprentissages de l'oral et de l'écrit et pour que se développent de nouvelles modélisations en DLE, qui apporteraient des réponses aux problématiques des programmes universitaires de LLC et viseraient au développement de réelles compétences translinguistiques et transculturelles. 


\subsection{Les travaux sur les stratégies et la métacognition}

Les recherches sur les stratégies d'apprentissage et la métacognition sont issues des d'études effectuées en psychologie cognitive. La psychologie cognitive s'intéresse aux mécanismes de construction et d'organisation des connaissances, et c'est grâce aux travaux d'O'Malley et Chamot (1990), qui ont établi une typologie des stratégies d'apprentissage pour la DLE, que s'est effectué le rapprochement entre cognitivisme et didactique des langues. Les stratégies sont considérées comme des opérations ou des étapes utilisées par un apprenant qui faciliteront l'acquisition, la mise en mémoire, la récupération ou l'utilisation de l'information. Elles sont classées en trois catégories principales : les stratégies cognitives, qui sont spécifiques par rapport à une tâche donnée et supposent la manipulation directe du matériel à apprendre; les stratégies métacognitives, qui sont d'ordre plus général, telles que la planification, l'identification de problème, etc. et sont applicables à de nombreuses tâches; et les stratégies socioaffectives, qui concernent l'interaction de l'apprenant avec d'autres personnes afin de faciliter les apprentissages. Les stratégies métacognitives relèvent de ce que la psychologue Ann Brown (1982) appelle « l'agent exécutif », elles sont capitales dans la conduite de l'apprentissage d'une tâche complexe, puisqu'elles impliquent des activités de planification, de contrôle, de vérification et de régulation.

En psychologie cognitive, une distinction importante est établie entre l'information "statique » en mémoire, les connaissances déclaratives (declarative knowledge: what we know about) et l'information « dynamique » en mémoire, les connaissances procédurales (procedural knowledge : what we know how to do), distinction qui sera reprise en DLE. Dans une optique d'intervention pédagogique, l'enseignement, selon les principes cognitivistes doit contribuer à faciliter l'encodage, la mise en mémoire et la récupération des informations et permettre un niveau suffisant d'automatisation de certaines connaissances pour conduire à la procéduralisation. Mais l'intervention doit également aider l'apprenant à construire de nouvelles connaissances à partir de ses connaissances initiales, à s'engager dans des activités de réflexion et d'analyse de sa propre cognition (la métacognition, sorte de métapensée ou «thinking about thinking », selon les termes de Flavell, 1979), et faciliter les activités de régulation de l'apprentissage. Ce sont ces propositions que nous avons regroupées sous l'appellation de "modèles métacognitifs », car elles reposent sur les prémisses des théories cognitives de l'apprentissage, mais mettent l'accent sur le rôle de l'enseignant et des co-apprenants dans des activités de médiation, d'étayage et de « métacognition partagée » (Paris \&Winograd, 1990), offrant ainsi des articulations particulièrement intéressantes avec la dimension sociale des apprentissages, comme nous allons le voir.

Ce que ces modèles d'intervention proposent, c'est d'articuler les notions-clés de tâche, de stratégies d'apprentissage, de métacognition et de régulation des apprentissages, dans des modélisations d'intervention didactique où les rôles des apprenants et de l'enseignant sont redéfinis. Dans un modèle métacognitif, le rôle de l'enseignant ne consiste plus seulement à proposer des pratiques répétées, assorties d'évaluations, mais à construire de "véritables objets d'apprentissage », pour reprendre les mots de Joachim Dolz et Bernard Schneuwly (1998). Il faut partir des connaissances initiales des apprenants, les activer, encourager les démarches de conscientisation, favoriser le développement par modelage des stratégies cognitives et métacognitives et ancrer cette démarche de conscientisation dans des séances de pratique de la tâche.

La tâche est ici un dispositif d'apprentissage structuré, qui répond à un objectif particulier, dont les prérequis ont été analysés par l'enseignant, qui demande la mise en œuvre de démarches spécifiques et dont le but essentiel est de faciliter l'acquisition, la manipulation ou la consolidation des éléments à apprendre. La notion de tâche n'est pas nouvelle, elle existait déjà dans l'AC, mais il s'agit ici d'en proposer une gestion qui s'appuie sur les apports théoriques concernant les processus cognitifs et métacognitifs. En effet, dans le cadre de l'AC, les tâches sont généralement des constructions pédagogiques qui ciblent la communication et permettent aux apprenants « de comprendre, de manipuler, de produire et d'interagir dans la langue cible, alors que leur attention se porte principalement sur le sens plutôt que sur la forme » (Nunan, 1989, p. 10). Certains auteurs, dont Ellis (2003), ont fait avancer la notion en proposant de classer les tâches en deux catégories : les tâches non ciblées (unfocused), dont l'objectif principal est de permettre les interactions entre apprenants et qui sont de nature uniquement 
communicatives, et les tâches ciblées (focused), dans lesquelles l'enseignant attire l'attention des apprenants sur les formes linguistiques visées et qui seront nécessaires pour le bon déroulement de l'activité. Or, la recherche actuelle en psycholinguistique, comme le souligne Ellis, semble indiquer que les tâches non ciblées sont moins efficaces que les tâches ciblées, en raison notamment des compétences stratégiques des apprenants, qui ont tendance à se reposer sur l'emploi de structures déjà acquises lors d'activités purement communicatives.

Reprise dans le cadre des modèles métacognitifs, la tâche est ciblée : par sa structure même, elle exige la manipulation et la maitrise graduelle du matériel linguistique à apprendre et a un ancrage dans la réalité. Car pour être efficace, une tâche doit proposer des activités porteuses de sens, et déboucher sur un « résultat» (prise de décision, résolution de problème, production d'un message, etc.). Elle diffère en cela de l'exercice, qui exige une manipulation plus étroite et souvent décontextualisée des éléments linguistiques. La tâche ciblée permet de présenter le matériel à apprendre, de guider l'attention de l'apprenant à la fois sur la forme et sur le sens (focus on form and meaning), et donne des opportunités de pratiquer, de procéduraliser les connaissances et de renforcer les apprentissages.

Ce que les modèles métacognitifs apportent à la notion de tâche " ciblée », c'est un cadre théorique, dont les résultats sont étayés par de nombreuses recherches sur les processus cognitifs et métacognitifs, qui va guider l'enseignant dans le traitement didactique, en fonction de ses objectifs. Il ne s'agira pas de revenir à une structure canonique de leçon, mais de comprendre un certain nombre de principes généraux liés à l'apprentissage pour se forger des outils professionnels adaptables à des situations différentes, qui ne se limiteront pas aux apprentissages de nature linguistique et pourront être mis en œuvre pour des objectifs divers. Il y aura donc une intégration pédagogique de la «forme» et du «sens», ainsi qu'une intervention didactique particulière de la part de l'enseignant, qui vise à catalyser et à renforcer les apprentissages.

Dans un modèle métacognitif, le traitement didactique d'une tâche s'effectue en plusieurs étapes. Dans un premier temps, dans un souci d'organisation et de structuration des connaissances, après avoir analysé les pré-requis de la tâche, l'enseignant propose des activités qui permettent de guider l'attention des apprenants vers les éléments qu'ils doivent acquérir ou repérer (stratégies d'attention sélective). Il activera leurs connaissances antérieures (stratégies d'élaboration, ou mise en relation des connaissances antérieures et de l'information nouvelle, des différentes parties de l'information nouvelle, élaboration d'associations, etc.), les aidera à mettre en œuvre des activités de planification, à faire appel à des stratégies d'inférence. Dans un deuxième temps, l'enseignant engagera également les apprenants dans des activités destinées à favoriser la métacognition et à modifier les croyances et représentations erronées que ceux-ci peuvent avoir et qui peuvent freiner les apprentissages.

Pour les tenants de l'approche métacognitive, en effet, au départ, les apprenants ont un certain nombre de représentations, de connaissances et de croyances au sujet de la tâche à effectuer, au sujet de la meilleure façon de l'effectuer, mais aussi sur eux-mêmes en tant qu'apprenants: ce sont leurs connaissances métacognitives, qui sont faillibles et verbalisables (Flavell, 1979). Ils ont aussi un certain nombre de connaissances déclaratives sur les contenus et domaines d'étude, que les activités vont chercher à faire émerger, pour des modifications ou remises en question éventuelles.

Les apprenants/novices s'engagent donc dans la tâche et sont confrontés aux difficultés de son exécution. Bénéficiant du guidage et de l'étayage de l'enseignant/expert (Wood, Bruner, \& Ross, 1976), dont le rôle est capital dans le processus, ils effectuent alors, grâce à la verbalisation, un recul sur leurs pratiques, prennent conscience de leur approche et se posent la question de l'adéquation des stratégies à la tâche. Ce processus de distanciation, accompagné de possibilités d'autoévaluation, leur permet à la fois d'enrichir leurs connaissances métacognitives et d'améliorer leurs capacités à exécuter la tâche. Il permettrait également une prise en charge accrue de l'apprentissage et favoriserait l'autorégulation.

Il est important de souligner que la prise de conscience, dans cette proposition, n'est pas seulement une démarche individuelle soutenue par l'enseignant. La conscientisation ou « métacognition partagée », est en effet, selon la définition de Paris et Winograd, une démarche collective: «Knowledge about thinking that can be shared among people» (1990, p. 25). L'accent est donc mis sur la dimension sociale des 
apprentissages et sur les réflexions collectives destinées à enrichir les connaissances métacognitives des apprenants. Les processus de médiation sont pluriels et co-construits et émergent des activités. Ces propositions sont particulièrement intéressantes en DLE pour les activités de production écrite, auxquelles on va relier des activités de lecture analytique et de "pratique interprétative ", en posant ainsi des jalons pour des modélisations didactiques nouvelles en DLE, qui permettront de traiter les problématiques des niveaux intermédiaires et avancés de l'apprentissage linguistique, quelque peu négligés par l'approche communicative $(\mathrm{AC})$. Le recours à ce type de modélisation ne se limite cependant pas à ces niveaux : une bonne maîtrise des notions permet à l'enseignant de concevoir des tâches d'apprentissage qui ciblent l'utilisation de certaines stratégies en fonction des compétences visées. On peut ainsi, à l'instar de Vandergrift (2004), proposer des activités de compréhension orale et jouer sur des approches ascendantes (bottom-up) ou descendantes (top-down), mettre en place des taches de production orale orales ou écrites, et varier les objectifs et les supports. L'interêt du modèle, dans l'optique de la formation des futurs enseignants est d'apporter une cohérence globale tout en offrant un cadre très souple applicable à des situations très diverses.

Les modèles métacognitifs, nous venons de le voir, s'intéressent à la relation dynamique entre les connaissances métacognitives des apprenants et leurs capacités à réguler leur propre apprentissage. Mais ils rejoignent également les conceptions vygotskiennes de l'appropriation des connaissances, puisqu'ils accordent une place centrale à la médiation des processus cognitifs à travers le traitement didactique et les interactions, dans lesquelles le langage joue un rôle central. C'est cette convergence que nous souhaitons mettre en évidence à travers l'exemple de propositions issues des travaux interactionnistes et socioculturels concernant les apprentissages de la compréhension et de la production écrite.

\subsection{Les perspectives interactionnistes vygotskiennes}

Ce courant est basé sur les travaux théoriques du psychologue soviétique Lev Vygotsky (1934) et présente un intérêt particulier parce qu'il s'efforce de «saisir les faits langagiers au titre de traces de conduites humaines socialement contextualisées» (Bronckart, 1996, p. 21). Mais il va plus loin que certains des courants interactionnistes car il se fonde sur l'idée que l'interaction sociale est constitutive du développement des fonctions psychiques supérieures. Bien que ce courant ne constitue pas une approche unifiée, comme nous l'avons mentionné, nous regrouperons pour notre argumentation les divers travaux qui en émanent, (théorie socioculturelle et interactionnisme d'inspiration vygotskienne) et qui posent le postulat du rôle constitutif des interactions et des conditions sociales qui les régissent pour les produits et les processus d'apprentissage (Doehler, 2000).

Dans le cadre d'inspiration vygotskienne, la langue n'est pas conçue comme un simple outil, c'est « d'abord et avant tout une activité discursive, intimement liée à l'activité humaine dont elle constitue à la fois le reflet et le principal instrument» (Bronckart, 1985, p. 8). C'est aussi le véhicule privilégié de l'expression symbolique, ancrée dans une culture. Selon les termes du psycholinguiste genevois (Ibid., 2000), dans la vision vygotskienne, « l'activité langagière est une activité de mise en sens du monde, elle permet aux êtres humains de se le représenter, de le réfléchir, de le modifier. Elle rend possibles les formes de coopération en même temps qu'elle en résulte » (Bronckart, 2000). Le langage est ainsi conçu comme «instrument majeur de l'activité humaine, sous-tendu et organisé par les conditions et les motivations mêmes de cette activité » (Bronckart, 1985, p. 18).

Pour Vygotsky, c'est dans l'interaction que le langage intervient dans la formation de «mondes communs » (Clot, 2002, p. 89) " chargés de significations partagées et de références communes » (Vergnaud, 2002, p. 59). Par ses fonctions de représentation et de communication et par sa fonction symbolique et sémiotique, le langage occupe ainsi un rôle central dans la transmission et la création du culturel et l'approche des textes et discours produits par une culture donnée est essentielle dans la démarche d'apprentissage d'une langue étrangère.

En plaçant l'apprentissage et le développement au cœur des interactions sociales, le courant vygotskien s'efforce de penser l'apprenant dans ses capacités cognitives, mais aussi dans le réseau complexe de ses relations sociales, de ses processus d'interprétation et dans ses représentations du monde. Ainsi, 
l'apprenant n'est pas une simple boîte noire qui traite l'information, mais un sujet social, qui construit ses connaissances et établit des connexions signifiantes en fonction de ses représentations, de ses connaissances initiales et du contexte dans lequel il évolue, dans une dynamique constante. Cette conception de l'apprentissage est compatible avec les apports des recherches sur l'apprenant effectuées en psychologie cognitive et sociocognitive, mais en élargit la vision, en permettant une articulation entre le cognitif, le social et le culturel.

La culture est en effet conçue comme étant à la fois l'élément appris du comportement humain par enculturation (ensemble des opérations par lesquelles les sujets s'approprient la culture de leur groupe, une dynamique qui est une dimension de la socialisation) et l'immersion des individus dans un vaste système d'entités matérielles et symboliques (issus des activités collectives) se formant et évoluant tout au long de leur existence et qu'ils contribuent à modifier. Dans le cadre d'inspiration vygotskienne, l'apprentissage et le développement cognitif sont considérés comme étant liés de façon indissociable à la culture, comme nous allons le voir avec la notion centrale de « systèmes de médiation » entre l'homme et l'environnement.

Pour Vygotsky, «le fait central de notre psychologie est le fait de la médiation » (Vygotsky, 1982, p. 166, cité par Wertsch, 1985, p. 139). Dans la vision vygotskienne, en effet, l'ensemble des entités intermédiaires entre l'individu et le monde qui l'entoure relève de ce que le psychologue soviétique appelle «les systèmes de médiation ». Parmi ces instruments [qui sont sociaux par nature et non pas organiques ou individuels] figurent entre autres " le langage, les diverses formes de comptage et de calcul, les moyens mnémotechniques, les symboles algébriques, les œuvres d'art, l'écriture, les schémas, les diagrammes, les cartes, les plans, tous les signes possibles (Vygotsky, 1931, dans Bronckart et al. 1985, p. 39). Selon Vygotsky, les instruments produits historiquement par la culture transforment les processus mentaux de l'individu, ce qui signifie que «la forme du fonctionnement individuel est profondément influencée par ses origines sociales » (Wertsch, 1985, p.143).

Ce qui est avancé ici, c'est une sociogenèse des capacités cognitives, dans laquelle les systèmes sémiotiques (compris comme l'ensemble des signes et symboles culturellement et historiquement ancrés, dont le langage) jouent un rôle majeur puisqu'ils contribuent à la structuration et à l'organisation des fonctions psychiques supérieures. Vygotsky établit en outre une distinction entre les systèmes de signes (qu'il nomme instruments psychologiques) «au service d'une gestion d'ensemble des activités humaines » (Bronckart, 2004, p. 2) et les instruments « au service de la réalisation concrète [de celles-ci] (qu'il appelle outils)» (Ibid., p. 2). Dans cette perspective, instruments psychologiques et outils (objets et développements technologiques) constituent un vaste système d'entités intermédiaires entre l'environnement et l'homme, que celui-ci assimile, s'approprie, oriente vers lui-même, et qu'il modifie, alors même que ces systèmes infléchissent son comportement et développent ses capacités, dans un processus dialectique. Ces outils culturels, dans leurs immenses gammes, sont donc « des extensions de l'homme », (Mac Luhan, 1964), c'est-à-dire « des prolongements et des amplificateurs des capacités humaines » (Ivic, 2000, p. 6). Cette vision de la culture, indissociable des processus d'apprentissage et de développement, offre une base théorique particulièrement fertile pour explorer les articulations entre le cognitif, le linguistique, le social et le culturel et envisager l'élaboration de démarches méthodologiques qui permettraient de les traiter de façon conjointe.

Pour revenir à nos préoccupations, qui concernent une certaine harmonisation des approches et démarches méthodologiques dans nos cursus pour réduire les fractures actuelles, il nous semble que le rapprochement entre les propositions issues des modèles métacognitifs et celles qui proviennent du courant d'inspiration vygotskienne peuvent nourrir la réflexion pour l'élaboration de nouvelles modélisations didactiques en matière de compréhension et de production écrite qui aiderait à conceptualiser les liens entre cognition, interactions sociales et apprentissage linguistiques et culturels. Ceci permettrait d'adopter une démarche plus intégrative entre les différents niveaux du cursus et favoriserait la mise en place de formations plus pertinentes pour les futurs enseignants-chercheurs, en proposant un cadre théorique suffisamment large pour que soient imbriqués les volets linguistiques, cognitifs et socioculturels et que se développe une véritable didactique des textes en LE pour les cursus universitaires dès les premiers niveaux. 


\subsection{Objectif littératie(s) : pour une approche intégrative de la lecture et de la production écrite}

Nous avons vu le rôle majeur des interactions sociales et de l'appropriation des instruments psychologiques dans la vision vygotskienne du développement des capacités cognitives supérieures. Pour Vygotsky, «l'éducation ne fait pas qu'exercer une influence sur un certain processus évolutif; elle restructure de manière fondamentale toutes les fonctions du comportement » (Vygotsky, 1930, dans Bronckart et al. 1985, p. 45). Le psychologue soviétique, qui au delà de ses activités de recherches théoriques a été très engagé dans des actions pédagogiques, considère en effet que «l'éducation ne se réduit pas à l'acquisition d'un ensemble d'informations, elle est l'une des sources du développement », (Ivic, 2000, p. 11). Le rôle de l'enseignement systématique et structuré est central pour le développement (enfant/adulte ou novice/expert) et la fonction de l'enseignant comme médiateur des apprentissages y est fondamentale.

La conception vygotskienne de l'enseignement, tout comme celle issue des modèles métacognitifs accorde beaucoup d'importance aux activités de médiation de l'enseignant (comprises ici comme l'ensemble des aides ou des supports qu'une personne peut offrir à une autre personne en vue de lui rendre plus accessible un savoir quelconque) ${ }^{2}$ et à la création d'un environnement porteur de sens pour l'apprenant, car les recherches indiquent que celui-ci construit ses connaissances de façon plus efficace s'il est engagé personnellement. Les interactions ne sont plus simplement comme dans le cadre de l'AC, un simple déclencheur des processus cognitifs, elles sont constitutives des processus d'apprentissage et de développement et «constructives des savoirs et savoir-faire langagiers et de l'identité même de l'apprenant » (Doehler, 2000).

Dans les modèles métacognitifs, tout comme dans les travaux d'inspiration vygotskienne portant sur la relation apprentissage/enseignement, l'accent est mis sur les réflexions collectives destinées à enrichir les connaissances métacognitives des apprenants, grâce à la création d'un « champ de tension » (Schneuwly, 2008) potentiellement transformateur. Par l'importance accordée à l'ancrage social des apprentissages et au langage comme instrument de médiation, au moyen duquel « la pensée se réorganise et se modifie », (Vygotsky, 1934/1997, p. 431), ces deux visions convergent et méritent d'être rapprochées pour que puisse se développer des cursus visant la littératie et qu'émergent de nouvelles formations pour les enseignants-chercheurs de LLC.

Dans ce cadre que nous qualifierons de sociocognitif en effet, les enseignants pourront s'attacher à « construire de véritables objets d'apprentissage », pour reprendre les termes de Joachim Dolz et de Bernard Schneuwly (Dolz \& Schneuwly, 1998), et proposer, dès les premiers niveaux, des séquences didactiques où il sera possible pour les apprenants de se poser des questions sur la façon dont les «textes» (oraux et écrits) sont produits et reçus dans des contextes culturels différents. Il s'agira d'encourager la réflexion critique sur les mécanismes d'élaboration et d'interprétation du sens, de sensibiliser les apprenants aux différentes dimensions de la communication, d'articuler les apprentissages linguistiques concernant les formes avec ceux concernant les significations, d'aider les apprenants à prendre conscience des liens entre les caractéristiques textuelles et les pratiques discursives.

La démarche reposera sur la notion de genre discursif, issue des réflexions de Bakhtine (1977). Du point de vue didactique, Dolz et Schneuwly se basent en effet sur les propositions du philosophe russe, selon lesquelles les genres du discours sont des types d'énoncés relativement stables, émanant d'une communauté donnée, caractérisés non seulement par leur contenu thématique, mais aussi par la sélection opérée dans les moyens linguistiques, ainsi que par leur construction compositionnelle et dont on peut étudier les caractéristiques et les récurrences. Comme le soulignent ces auteurs, le travail sur les genres fournit « un cadre d'analyse des contenus, de l'organisation de l'ensemble du texte et des séquences qui le composent, ainsi que des unités linguistiques et des caractéristiques spécifiques à la textualité » (Dolz, Schneuwly, 1998, p. 65).

Le recours à la notion de genre permet de modéliser des séquences didactiques visant à la sensibilisation aux caractéristiques textuelles, à la réflexion collective sur les différents aspects de la mise en texte, et à l'appropriation d'un certain nombre de moyens linguistiques, ainsi qu'à un travail approfondi 
d'interprétation. Les dimensions culturelles des apprentissages sont traitées au cours de ces séquences de réflexion collective qui visent à développer chez les apprenants une conscience critique des relations entre textes, conventions discursives et contexte culturel historique et social. Les perspectives sont ici clairement vygotskiennes, puisque les activités de médiation et d'interactions sont le moteur du développement. On retrouve aussi dans ces propositions l'idée de la "pédagogie de pratique interprétative » de Kramsch $(1993,1995)$ et des tenants du courants de la littératie. La mise en évidence de l'imbrication entre le textuel et le discursif, à partir de textes simples dans les premiers niveaux, vers des textes plus complexes dans les niveaux intermédiaires et avancés aurait un double intérêt. D'une part, cette démarche faciliterait l'accès aux textes littéraires et l'appropriation progressive par les apprenants de compétences de lecture et écriture en LE ; d'autre part, elle permettrait aux futurs enseignants d'envisager le cursus et leurs propres pratiques professionnelles dans un cadre théorique plus cohérent, réduisant en cela les fractures actuelles.

Du point de vue cognitif, la lecture attentive de textes grâce à l'outil que constitue la notion de genre discursif aiderait à la constitution par l'apprenant de «schémas textuels » qui faciliterait la gestion des opérations de traitement impliquées et diminuerait la charge cognitive. Les travaux effectués en psycholinguistique ont démontré en effet que la production écrite en langue étrangère impliquait la maitrise et l'orchestration d'un très grand nombre d'opérations complexes à très forte charge cognitive, qui présentent de redoutables difficultés pour les apprenants (on sait par exemple que l'empan de mémoire est systématiquement plus faible en LE qu'en langue maternelle (LM), ce qui signifie une plus grande difficulté dans les opérations de planification). On sait aussi que la transposition des processus dits de « haut niveau », qui concernent la macrostructure textuelle (ou principes d'organisation du discours) ne se fait pas de façon très aisée de la LM vers la LE (Gaonac'h \& Larigauderie, 2000, p. 245).

Le travail de sensibilisation aux différentes dimensions d'un genre donné, (situation de communication, organisation interne du discours, caractéristiques linguistiques pour traiter les deux dimensions précédentes) à travers la lecture analytique collective pourrait contribuer à une meilleure appropriation et automatisation des processus de bas niveau (traitement phonologique, lexical, syntaxique), ainsi qu'à la constitution des schémas cognitifs qui faciliterait les opérations de traitement et d'allocations de ressources cognitives et faciliteraient la mise en œuvre des processus de haut niveau. Les principes de gestion des modèles métacognitifs sont utiles et pertinents pour la conduite de telles tâches et on voit ici nettement émerger les convergences entre les deux courants.

\section{Perspectives}

Les démarches que nous venons de décrire présentent donc un grand nombre de points intéressants pour l'élaboration de nouveaux cursus plus unifiés dans nos programmes de LLC. La mise en regard et l'articulation des différents concepts et courants théoriques que nous avons évoqués dans le cadre « d'une cognition située " (Mondada, 2000), où les textes relevant de genres discursifs divers retrouvent leur place dans des activités de pratique analytiques et interprétatives permet d'envisager l'émergence de nouvelles méthodologies, et la création de formations professionnelles pour les futurs enseignantschercheurs dans lesquelles les volets linguistiques, historico-culturels et sociaux ne seraient plus dissociés, pour une approche des apprentissages des langues, cultures et littératures étrangères qui ne seraient plus disjoints. Dans ces nouvelles pratiques, lecture et écriture ne seraient plus des activités périphériques mais « un noyau important où le linguistique, le culturel et la pensée convergent» (Kern, 2000).

Pour nous, les apports théoriques sur les stratégies d'apprentissage et la métacognition partagée et les travaux relevant de conceptions vygotskiennes du développement dans l'apprentissage sont complémentaires par les éclairages qu'ils apportent sur les rôles respectifs des apprenants et de l'enseignant au sein de la configuration didactique. Le rapprochement de ces paradigmes permettrait d'envisager une meilleure continuité dans les pratiques pédagogiques tout au long d'un cursus. Dans un cadre théorique de la cognition située plus solide et plus cohérent, l'articulation avec la réflexion linguistique dans la diversité de ses courants (de la linguistique de la « langue », à celle du « cerveau » en 
passant par la pragmatique, la linguistique sociale, interactionniste ou discursive, pour ne citer que quelques exemples) pourrait continuer à nourrir la DLE. Les fractures entre «cursus de langue » et « cursus de littérature » seraient estompées et un dialogue constructif pourrait alors voir le jour, sur la base de formations professionnelles nouvelles.

Il est maintenant essentiel que les divers niveaux d'analyse se nourrissent mutuellement et que se multiplient les recherches interdisciplinaires et les tentatives d'intégration et d'articulation des divers paradigmes de recherche, pour que puisse avancer la réflexion didactique et que se renouvellent et s'enrichissent les approches et les pratiques de classe dans des cursus plus riches et plus unifiés, aptes à répondre aux objectifs de l'enseignement supérieur de langues étrangères, littérature et culture.

\section{Références bibliographiques}

Adam, J. M. (1999). Linguistique textuelle. Des genres de discours aux textes. Paris : Nathan Université.

Bakhtine, M. (1977). Le Marxisme et la philosophie du langage. Paris : Editions de Minuit.

Bakhtine M. (1984). Esthétique de la création verbale. Paris : Gallimard.

Bronckart, J.P. (1985). Le fonctionnement des discours: un modèle psychologique et une méthode d'analyse. Paris: Delachaux et Niestlé.

Bronckart, J.P. (1996). Activité langagière, texte et discours : pour un interactionnisme socio-discursif. Lausanne : Delachaux et Niestlé.

Bronckart, J.P. (2000). "Les processus de construction de la personne dans la perspective de l'interactionnisme sociodiscursif", Séminaire ARC Langue(s) et identité(s) collective (s) du jeudi 6 avril 2000, Université de Genève.

Bronckart, J. P. (2004). "La médiation langagière, son statut et ses niveaux de réalisation ”, in R. Delamotte-Legrand (Ed.), Les médiations langagières, volume 2. Rouen : Publications de l’Université de Rouen.

Bronckart, J.P. John Steiner, V., Panofsky, C.P., Piaget, J., Schneuwly, B., Vygotsky, Wertsch, J.V. (1985). Vygotsky aujourd'hui. Neuchâtel-Paris : Delachaux \& Niestlé.

Breen, M. (2001). Learner Contributions to Language Learning. Harlow: Pearson Education.

Brown, A. (1982). "Inducing strategic learning from texts by means of informed, self-control training", Topics in Learning and Learning Disabilities, 2(1), pp. 1-17.

Chamot, A. (2005). "Language Learning Strategy Instruction: Current Issues and Research", Annual Review of Applied Linguistics, 25, 112-130.

Clot, Y. (2002). Avec Vygotski. Paris : Editions de la Découverte.

Cuq, J.P., Gruca, I. (2005). Cours de didactique du français langue étrangère. Grenoble : Presses Universitaires de Grenoble.

Doehler, S. (2000). “Approches interactionnistes des langues étrangères : concepts, recherches et perspectives”, Aile 12.

Dolz, J., Schneuwly, B. (1998). Pour un enseignement de l'oral: initiation aux genres formels à l'école, Paris: ESF.

Ellis, R. (2003). Task-based Language Learning and Teaching. Oxford : Oxford University Press.

Flavell J.H. (1979): "Metacognition and Cognitive Monitoring: A New Area of Cognitive Development Inquiry", American Psychologist 34 (10), pp. 906-911.

Flower, L. (1994). The Construction of Negociated Meaning : A Social Cognitive Theory of Writing. Carbondale, IL : Southern Illinois University.

Gaonac'h, D., Larigauderie, P. (2000). Mémoire et fonctionnement cognitif. Paris : Armand Colin.

Ivic, I. (2000). "Lev. S. Vygotsky”, Perspectives, 24 (3/4), 793-820.

Kern, R. (2000). Literacy and Language Teaching. Oxford : Oxford University Press.

Kramsch, C. (1993). Context and Culture in Language Teaching. Oxford: Oxford University Press. 
Kramsch, C. (1995). "Rhetorical Models of Understanding", in T. Miller (Ed.), Functional Approaches to Written Texts: Classroom Application. The TESOL France Journal, 61-78.

Lantolf, J., Appel, G. (1994). Vygotskian Approaches to Second Language Learning. Wesport, Ct: Ablex.

Lantolf, J. (Ed.). (2000). Sociocultural Theory and Second language Learning. Oxford: Oxford University Press.

Mac Luhan (1964). Understanding Media. The extensions of Man. New York : Mac Graw \& Hill.

Maingueneau, D. (2007). Analyser les textes de communication. Paris: Armand Colin.

Mangenot, F. (1998). “Outils textuels pour l'apprentissage de la lecture et de l'écriture en L1 et en L2.”, Pratiques discursives et acquisition des langues étrangères, 27-38.

Moirand, S. (1990). Enseigner à communiquer en langue étrangère. Paris : Hachette.

Mondada, L., Doehler, P.S. (2000). “Interaction sociale et cognition située : quels modèles pour la recherche sur l'acquisition des langues ?, Aile 12.

Newman, F., Holzman L. (1993). Lev Vygotsky : revolutionary scientist. London : Routledge.

Nunan, D. (1989). Designing Tasks for the Communicative Classroom. Cambridge: Cambridge University Press.

O’Malley J.M., Chamot, A. (1990). Learning Strategies in Second Language Acquisition. Cambridge: Cambridge University Press.

Paris, S.G., Winograd P. (1990). "How Metacognition Can Promote Academic Learning and Instruction”, in Jones B.F. \& L. Idol (Eds), Dimensions of Thinking and Cognitive Instruction, Hillsdale, NJ. : Erlbaum Associates, pp.15-51.

Peytard, J. (1995). Mikhail Bakhtine. Dialogisme et analyse du discours. Paris : Bertrand-Lacoste.

Puren, C. (1999). La didactique des langues à la croisée des méthodes : Essai sur l'éclectisme. Paris: Didier.

Sagnier, C. (2010). Perspectives sociocognitives et socioculturelles sur la didactique des langues étrangères: de la théorie à la pratique. Manuscrit non-publié.

Sagnier, C. (2009). "Perspectives vygotskiennes sur la formation des futures enseignants de FLE", Les Cahiers de l'Adisfle, 21.

Sagnier, C. (2006). "Pour une intégration des paradigmes de recherche en didactique des langues", PArole.

Skehan, P. A cognitive Approach to Language Learning. Oxford: Oxford University Press.

Schneuwly, B. (2008). "Eléments d'histoire des 20 années passées et propositions conceptuelles pour la suite", in Brossard, M., Fijalkow, J. (Eds.), Vygotski et les recherches en éducation et didactique, Bordeaux : Presses Universitaires de Bordeaux, 19-34.

Swaffar, J.K., Arens M., H. Byrnes. (1991). Reading for meaning: An integrated Approach to Language Learning. Englewoods Cliffs, NJ: Prentice Hall.

Todorov, T. (1978). Les genres du discours. Paris : Seuil.

Vandergrift, L. (2004). “Listening to learn or Learning to listen ? ”, Annual Review of Applied Linguistics, 24, 3-25.

Vergnaud, G. (2002). “On n'a jamais fini de relire Vygotsky et Piaget”, in Clot Y. (Ed.). Avec Vygotski. Paris : Editions de la Découverte, 55-68.

Vygotsky (1935/1997). Pensée et langage. Paris: La Dispute.

Weinert, F., Kluwe, R. (1987). Metacognition, Motivation and Understanding. Hillsdale, NJ: Lawrence Erlbaum.

Wenden, A. (1999). "Metacognitive Knowledge and Beliefs in Language Learning”, System, 27(4).

Wertsch, J. (1991). Voices of the Mind. A sociocultural Approach to Mediated Action. Cambridge, Mass: Harvard University Press.

Wertsch, J. (1985). Vygotsky and the Social Formation of the Mind. Cambridge, Mass. : Harvard University Press.

Wertsch, J. (1985). Culture, communication and cognition: Vygotskian perspectives. Cambridge: Cambridge University Press. 
978-2-7598-0534-1, Paris, 2010, Institut de Linguistique Française

Didactique et enseignement, français langue maternelle, français langue seconde DOI $10.1051 / \mathrm{cmlf} / 2010166$

Wood, P, Bruner, J., Ross, G. (1976). "The role of tutoring in problem-solving", Journal of Child Psychology and Psychiatry, 17, 89-100.

\footnotetext{
${ }^{1}$ Modern Language Association Report on Foreign Languages and Higher Education, 2007.

${ }^{2}$ Raynal, F. Rieuner, A. (1998). Pédagogie : Dictionnaire des concepts clés. Apprentissages, formation, psychologie, Paris : ESF.
} 